\title{
Viscoelastic Effects in Tape-Springs
}

\author{
Kawai Kwok* and Sergio Pellegrino ${ }^{\dagger}$ \\ California Institute of Technology, Pasadena, CA 91125
}

\begin{abstract}
Following recent interest in constructing large self-deployable structures made of reinforced polymer materials, this paper presents a detailed study of viscoelastic effects in folding, stowage, and deployment of tape-springs which often act as deployment actuators in space structures. Folding and stowage behavior at different temperatures and rates are studied. It is found that the peak load increases with the folding rate but reduces with temperature. It is also shown that a load reduction of as much as $60 \%$ is possible during stowage due to relaxation behavior. Deployment behavior after significant load relaxation demonstrates features distinct from elastic tape-springs. It starts with a short dynamic response, followed by a quasi-static deployment, and ends with a slow creep recovery process. A key feature is that the localized fold stays stationary throughout deployment. Finite element simulations that incorporate an experimentally characterized viscoelastic material model are presented and found to capture the folding and stowage behavior accurately. The general features of deployment response are also predicted, but with larger discrepancy.
\end{abstract}

\section{Introduction and Background}

A continuing challenge in spacecraft applications is the packaging of large structures in the confined space of launch vehicles and the subsequent in-orbit deployment. Energy-storing structures provide a robust solution because they are able to self-deploy by releasing the strain energy stored during folding: reinforced polymer composites have become strong candidate materials because of their high thermal stability and specific stiffness. ${ }^{1}$ However, the inherent creep behavior of polymer matrix has limited the amount of deployment force and shape precision that the structure can achieve. An example of viscoelastic effects in deployable structures is the Mars Advanced Radar for Subsurface and Ionosphere Sounding (MARSIS) antenna on the Mars Express spacecraft. ${ }^{2}$ A reduction in deployment moment was observed in the folded composite booms forming the antenna after a long period of stowage and has been attributed to energy dissipation in the viscoelastic matrix. ${ }^{3}$ Since deployable structures are often stowed for a long time and subject to varying temperature environments, a reliable analysis needs to take into account the viscoelastic behavior of the material.

Effects of stowage have attracted interest recently. Previous work was primarily experimental, studying the recovery time ${ }^{4}$ and deployment dynamics ${ }^{5}$ of slotted composite booms after stowage. Limited analysis was proposed to model the structure to aid the understanding of the shape recovery process over time under general conditions. In a recent study ${ }^{6}$ we have correlated analytically the moment and shape evolution of a viscoelastic beam for different stowage durations and temperatures under quasi-static conditions. This paper aims to extend such investigations to tape-springs, which are routinely used as building blocks of deployable space structures ${ }^{7}$ to reveal the implications of viscoelastic effects.

The particular problem chosen is the folding, stowage and deployment of a thin tape-spring made of low density polyethylene (LDPE). A tape-spring is a thin shell with curved section, typically of uniform curvature and subtending an angle smaller than $180^{\circ}$, as shown in Figure 1. While the mechanical properties of solid polymers depends on their particular molecular structure that gives rise to the distinction between thermosets and thermoplastics, the phenomenological features of linear viscoelastic behavior of polymers are adequately represented by the same constitutive model provided that they are viewed under the correct temperature and time scale. The normalized stiffness variations over time (master curves) of LDPE and epoxies commonly

\footnotetext{
*Graduate Student, Graduate Aerospace Laboratories, 1200 E. California Blvd. MC 205-45. kwk5@caltech.edu

$\dagger$ Joyce and Kent Kresa Professor of Aeronautics and Professor of Civil Engineering, Graduate Aerospace Laboratories, 1200 E. California Blvd. MC 301-46. AIAA Fellow. sergiop@caltech.edu
} 
used in composites are similar provided that each curve is referenced to the respective glass transition temperature for that material. This remarkable feature is summarized in the time-temperature equivalence principle. Typical epoxies have glass transition temperatures above $100^{\circ} \mathrm{C}$, which add significant difficulty in experiments involving large displacements such as dynamic deployment. In choosing LDPE, whose glass transition is below $0^{\circ}, 8$ we can study viscoelastic effects in structures near room temperature and the results are indicative of thermosets above the glass transition, based on time-temperature equivalence.

It is well known that packaging of thin structures can be accomplished by exploiting geometric nonlinearities. In this paper, we study tape-springs folded by forming a local buckle and regard the process of folding-stowage-deployment as a continuous time-dependent event that takes into account the load and boundary condition histories. Experiments with detailed control of prescribed conditions against time are presented. The experimental results are compared with numerical simulations based on isotropic linear viscoelasticity. The current study is the first to provide the necessary techniques to incorporate viscoelastic material models in a global structural finite element analysis for deployable structures.

The paper is laid out as follows. Section II describes the fabrication of LDPE tape-springs and the experiments carried out for observing both the quasi-static folding and stowage, as well as dynamic deployment behavior. Section III reviews relevant aspects of the theory of linear viscoelasticity and describes the material characterization procedure used for the tape-spring. Section IV presents the finite element simulation techniques for modeling viscoelastic structures. Section V compares and discusses the experimental and numerical results. Section VI concludes the paper.

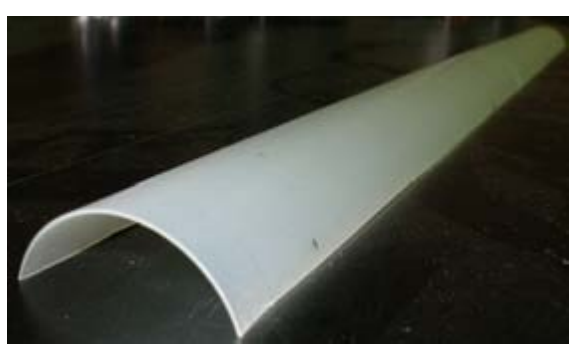

(a)

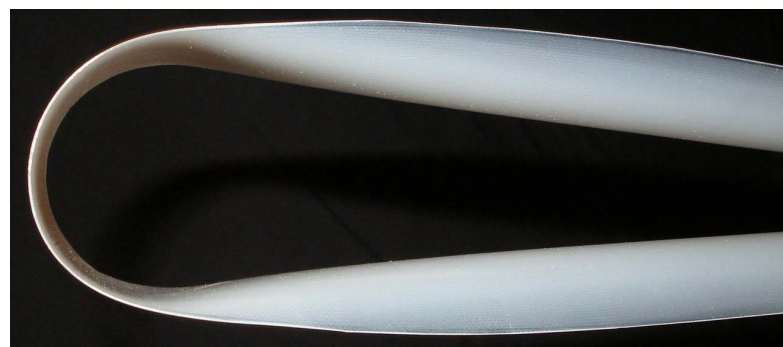

(b)

Figure 1: Photos of tape-spring: (a) deployed and (b) folded.

\section{Experiments}

Quasi-static folding and stowage, followed by dynamic deployment were investigated experimentally in sequence. Folding and stowage tests were conducted first, following a carefully designed procedure that could be executed under fully controlled conditions, with a known deformation history prior to deployment. Dynamic deployment experiments were then performed to observe the shape recovery process of tape-springs that had been subject to stowage.

All experiments were carried out on tape-springs with an inner diameter of $38 \mathrm{~mm}$, a nominal thickness of $0.73 \mathrm{~mm}$, and a subtended angle of $150^{\circ}$ fabricated from flat LDPE sheets through a thermal remolding process. In the process, a flat LDPE sheet was sandwiched between two PTFE release fabric layers, wrapped around a steel mandrel, restrained with heat shrink tape, and subject to a thermal cycle. Since LDPE is an uncrosslinked polymer, the processing temperature is its melting point. The assembly was heated to $120^{\circ} \mathrm{C}$, maintained at this temperature for 4 hours, and then allowed to cool to room temperature in 8 hours at a constant cooling rate inside an oven with a temperature control precision of $\pm 2^{\circ} \mathrm{C}$. The long heating and cooling periods allowed enough time for LDPE to recrystallize and to minimize the effect of physical ageing, respectively. They are important for achieving temporally stable mechanical properties in the remolded material. 


\section{A. Quasi-static Folding and Stowage}

Folding and stowage tests were performed by vertically compressing tape-spring specimens with a length of $272 \mathrm{~mm}$ in an Instron testing machine as shown in Figure 2. One challenge of conducting experiments with viscoelastic materials is the requirement of fully and precisely tracking the load and displacement measurements with respect to time. The exact deformation history needs to be controlled in order to reproduce the same response experimentally due to path dependence of the problem. Although tape springs can be easily folded by hand, a rather unusual fold-stow procedure was adopted for the present experiment, to achieve fully controlled load profiles and boundary conditions that can be measured simultaneously over time.

To allow the ends of the tape-spring to rotate freely through a large angle during folding, the connection between the end of the tape-spring and the load frame was established through point contact with thin aluminum plates attached to the Instron testing machine. Experiments at temperatures of $15^{\circ} \mathrm{C}$ and $22^{\circ} \mathrm{C}$ were conducted inside an environmental chamber (Instron Heatwave Model 3119-506) that has a temperature control precision of $\pm 1^{\circ} \mathrm{C}$.

During the test, a vertically downward displacement of $80 \mathrm{~mm}$ was applied to the tape-spring, which was then held in this configuration for $5000 \mathrm{~s}$. Two displacement rates, $1 \mathrm{~mm} / \mathrm{s}$ and $5 \mathrm{~mm} / \mathrm{s}$, were used. Load profiles over time were obtained and plotted in Figures 3 and 4.

As seen in Figure 3, the load drops significantly after buckling and continues to decrease even though the displacement is still decreasing. This is different from the post-buckling behavior of an Euler strut where the load vs. displacement response would have a slightly positive slope. There are two reasons for this difference, first the moment at the fold of an elastic tape spring is constant due to propagation of a local instability ${ }^{9}$ and second due to viscoelastic effects in the present case the moment actually decreases. Load relaxation takes place at the same time as buckle propagation and offsets the expected increase in force. It also shows that a higher load is required to fold the tape-spring at lower temperatures and faster rates. This behavior is typical of viscoelastic materials whose modulus increases with decreasing temperature and increasing rate.

Figure 4 plots the load on semi-log scale during the stowage period for the case of $1 \mathrm{~mm} / \mathrm{s}$ displacement rate. The linear shape of the curves on a log time axis implies that the reduction in load is exponential in time. After $5000 \mathrm{~s}$ the load has dropped to about half of its value at the end of folding.

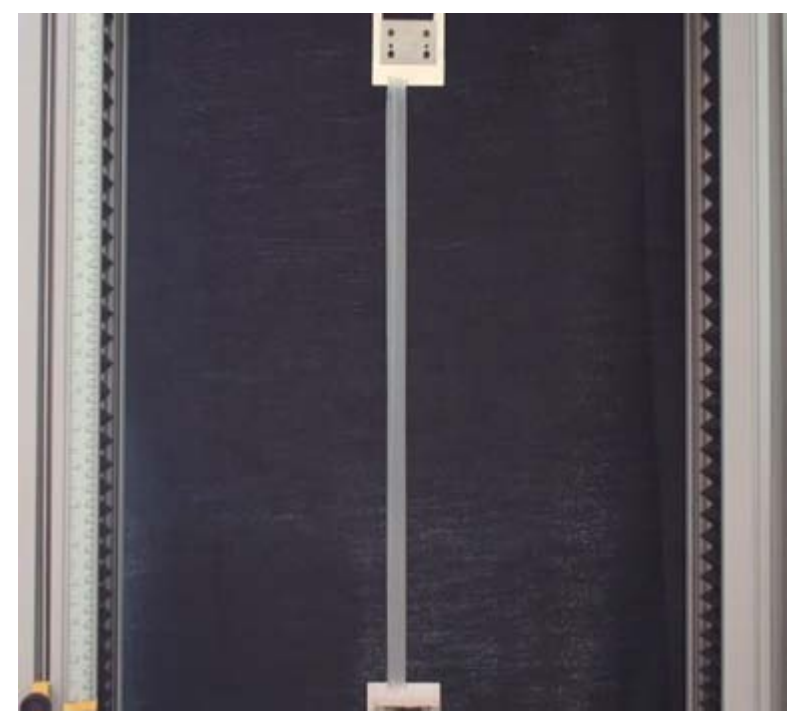

(a)

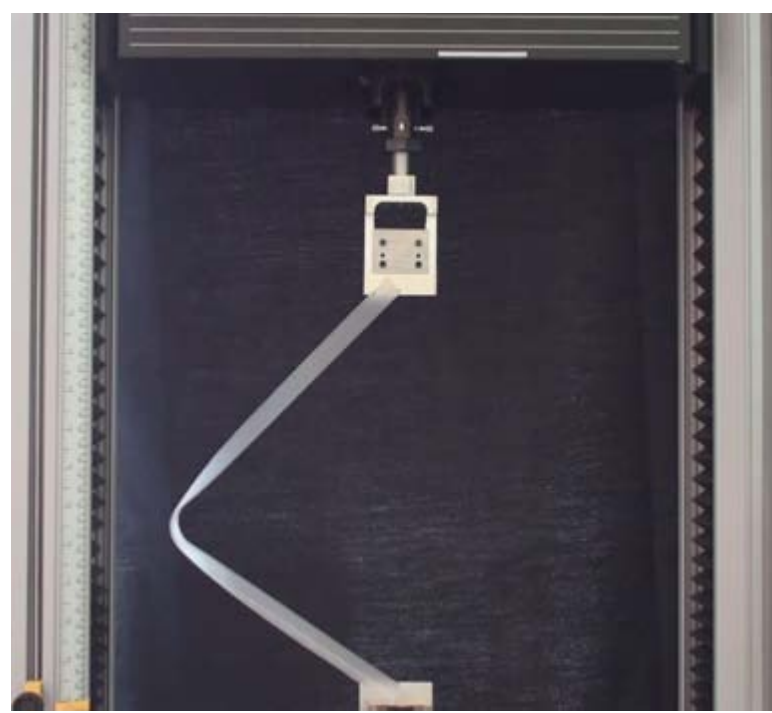

(b)

Figure 2: Folding and stowage test setup (environmental chamber not shown): (a) deployed (unstressed) configuration and (b) stowed configuration. 


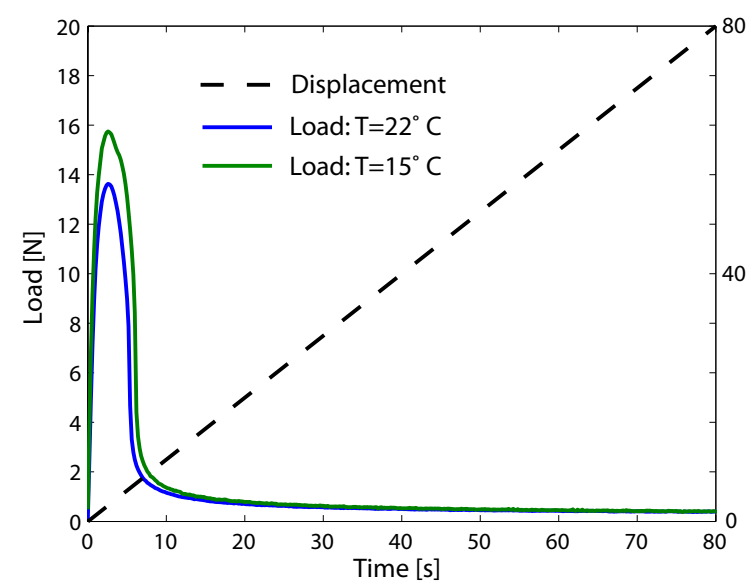

(a)

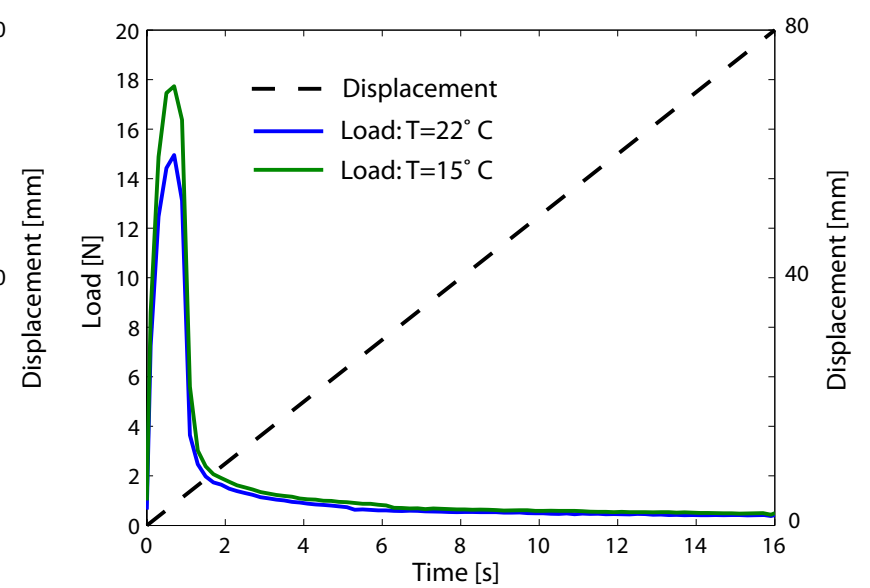

(b)

Figure 3: Load response during folding: (a) at a displacement rate of $1 \mathrm{~mm} / \mathrm{s}$ and (b) at a displacement rate of $5 \mathrm{~mm} / \mathrm{s}$.

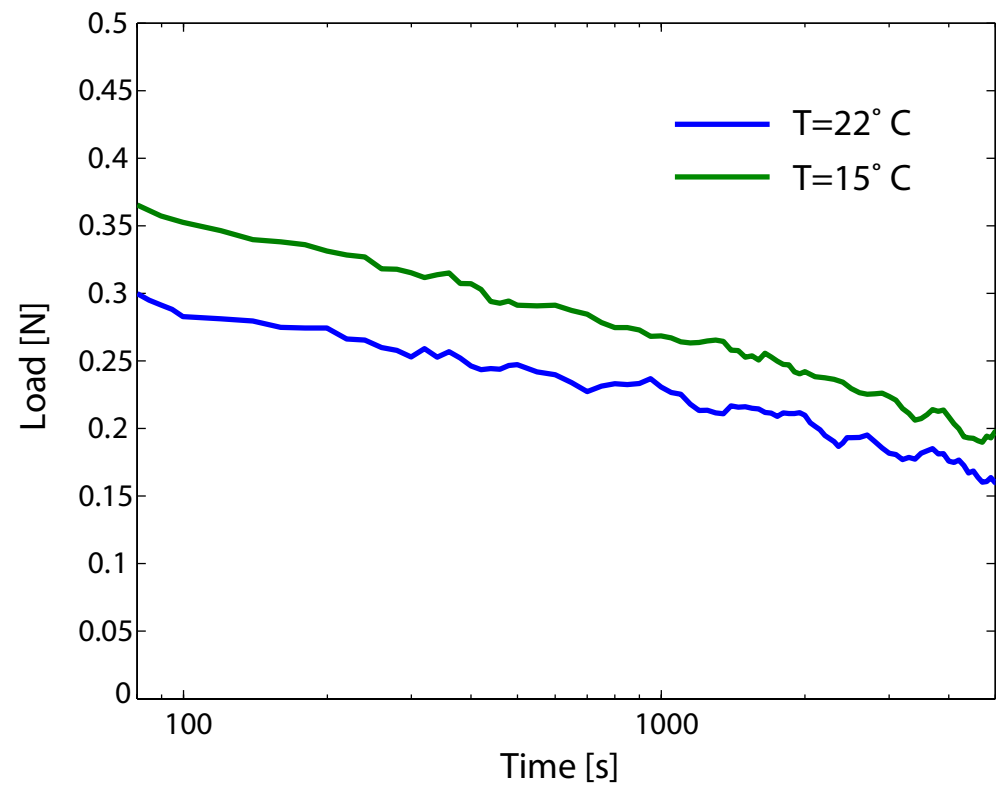

Figure 4: Load response in stowed configuration for a displacement rate of $1 \mathrm{~mm} / \mathrm{s}$. 


\section{B. Dynamic Deployment}

Deployment tests were conducted at room temperature on a tape-spring with a length of $398 \mathrm{~mm}$ using the experimental setup shown in Figure 5. The tape-spring was clamped on the bottom and positioned vertically. It was first folded to an angle of $87^{\circ}$ in $9 \mathrm{~s}$ and then held stowed for $983 \mathrm{~s}$. While in the stowed configuration, the force at the end of the tape-spring was measured by connecting it to a load cell through a string. Deployment was initiated by cutting the string at the end of stowing period. This experiment poses many of the general challenges that are encountered in applications of self-deployable structures, which relax during stowage and creep during deployment upon a sudden removal of displacement constraints.

To characterize the deformation of the tape-spring, a target point $\mathrm{P}$ near the free end was marked (Figure 5) and its lateral displacement $x_{p}$ was tracked during deployment. Large displacements were extracted from images taken using a high resolution camcorder with a frame rate of $30 \mathrm{fps}$. A laser displacement sensor (Keyence LK-G87) was used to measure the small values of $x_{p}$ that could not be measured accurately from the images.

Figure 6 shows the deployed shapes over time with different time steps. Their corresponding values of $x_{p}$ and points in time are marked in Figure 7, which includes three detailed views of the displacement response over time, each highlighting a particular feature. The deployment process can be divided into three stages with distinctive features. A dynamic response is seen during the first $5 \mathrm{~s}$, Figure $7(\mathrm{~b})$, and includes a low vibration magnitude with a period of about $0.8 \mathrm{~s}$ about a finite displacement that decreases with time. The next phase involves quasi-static deployment and occurs between $5 \mathrm{~s}$ and $55 \mathrm{~s}$, with the lateral displacement actually overshooting the deployed configuration by $11 \mathrm{~mm}$, Figure 7(c). Note that the fold location does not change during these first two stages: this behavior is different from that of a linear elastic tape-spring in which deployment is accompanied by the fold traveling towards the fixed end.$^{9}$ Finally, a slow creep recovery of the fold cross section leads to a nearly zero lateral displacement over a period of $3000 \mathrm{~s}$. It should be noted that the cross section recovery to its original geometry is only asymptotic.

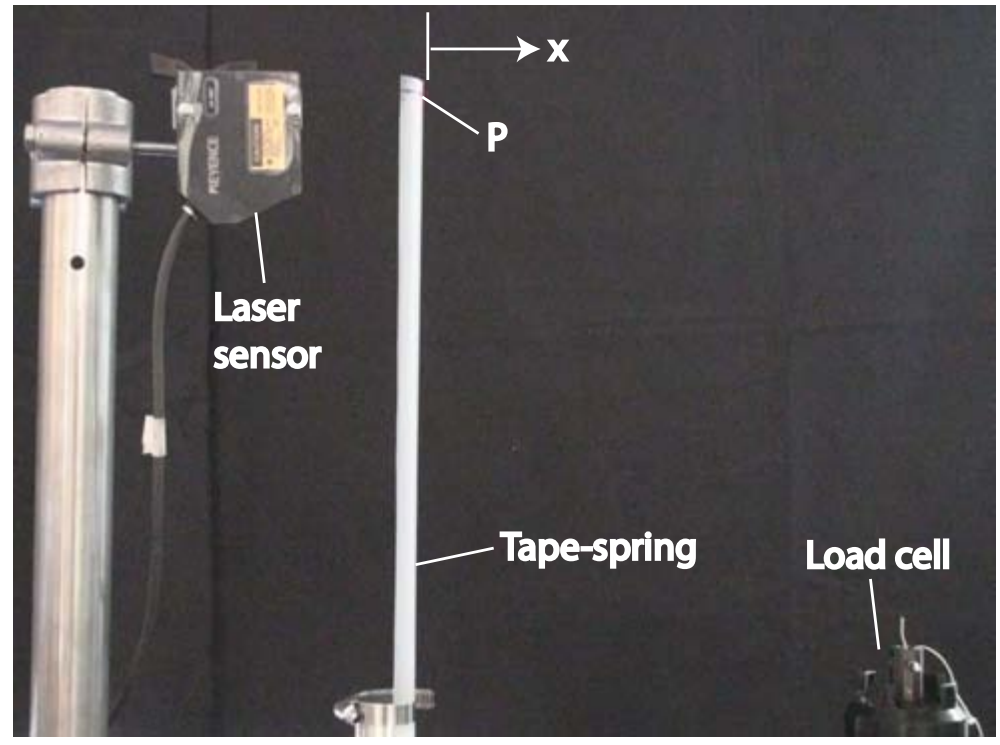

Figure 5: Experimental configuration for deployment test.

\section{Material Model}

The behavior observed in Section II has shown significant time-dependent effects. This section aims to provide the theoretical and experimental basis for modeling the material constitutive behavior to be incorporated into a structural finite element analysis. 


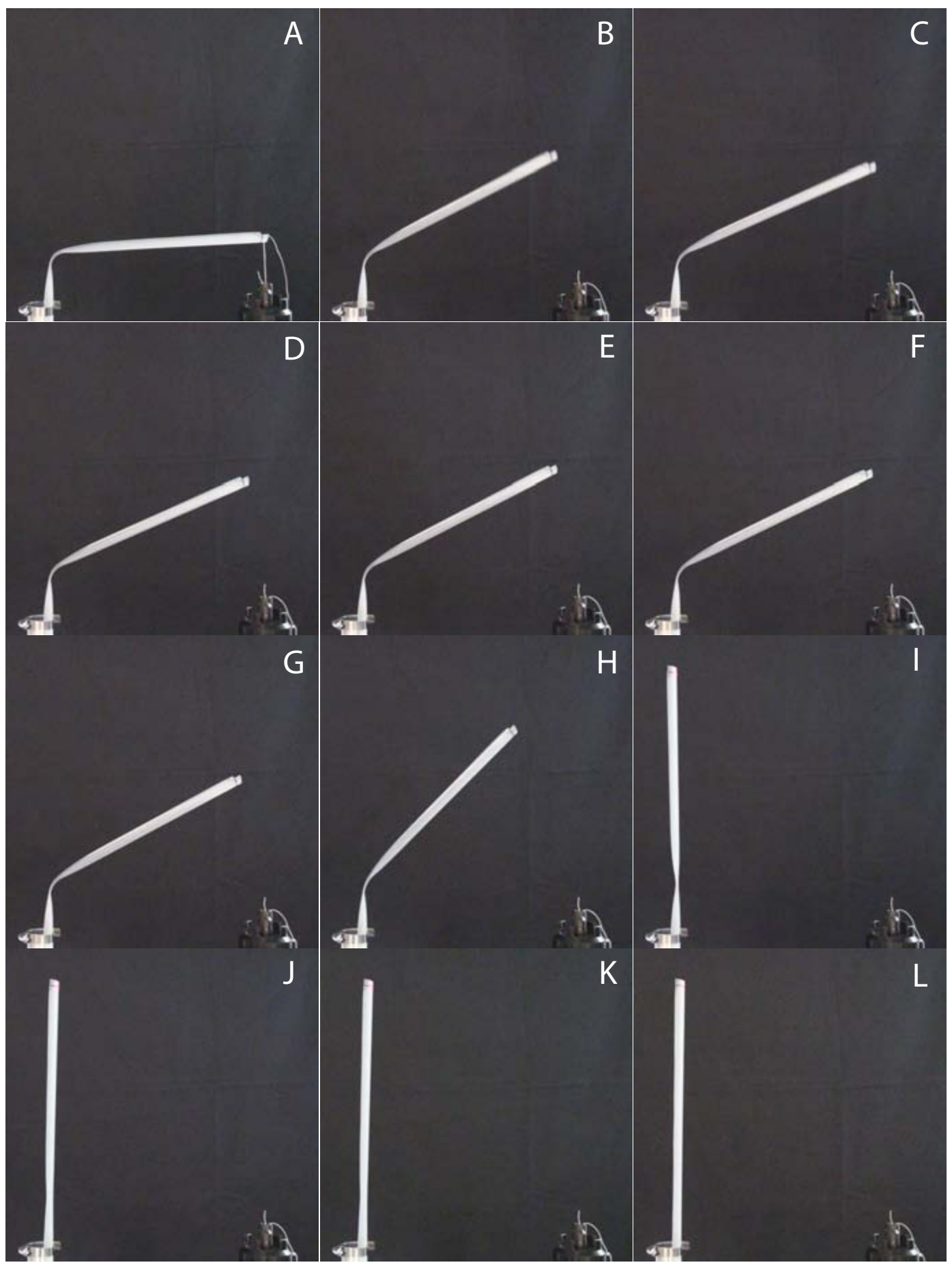

Figure 6: Deployment sequence: (A)-(F) from $0 \mathrm{~s}$ to $2.5 \mathrm{~s}$ in steps of $0.5 \mathrm{~s},(\mathrm{G})-(\mathrm{I})$ : from $5 \mathrm{~s}$ to $55 \mathrm{~s}$ in steps of $25 \mathrm{~s}$, and $(\mathrm{J})-(\mathrm{L})$ : from $1000 \mathrm{~s}$ to $3000 \mathrm{~s}$ in steps of $1000 \mathrm{~s}$. 


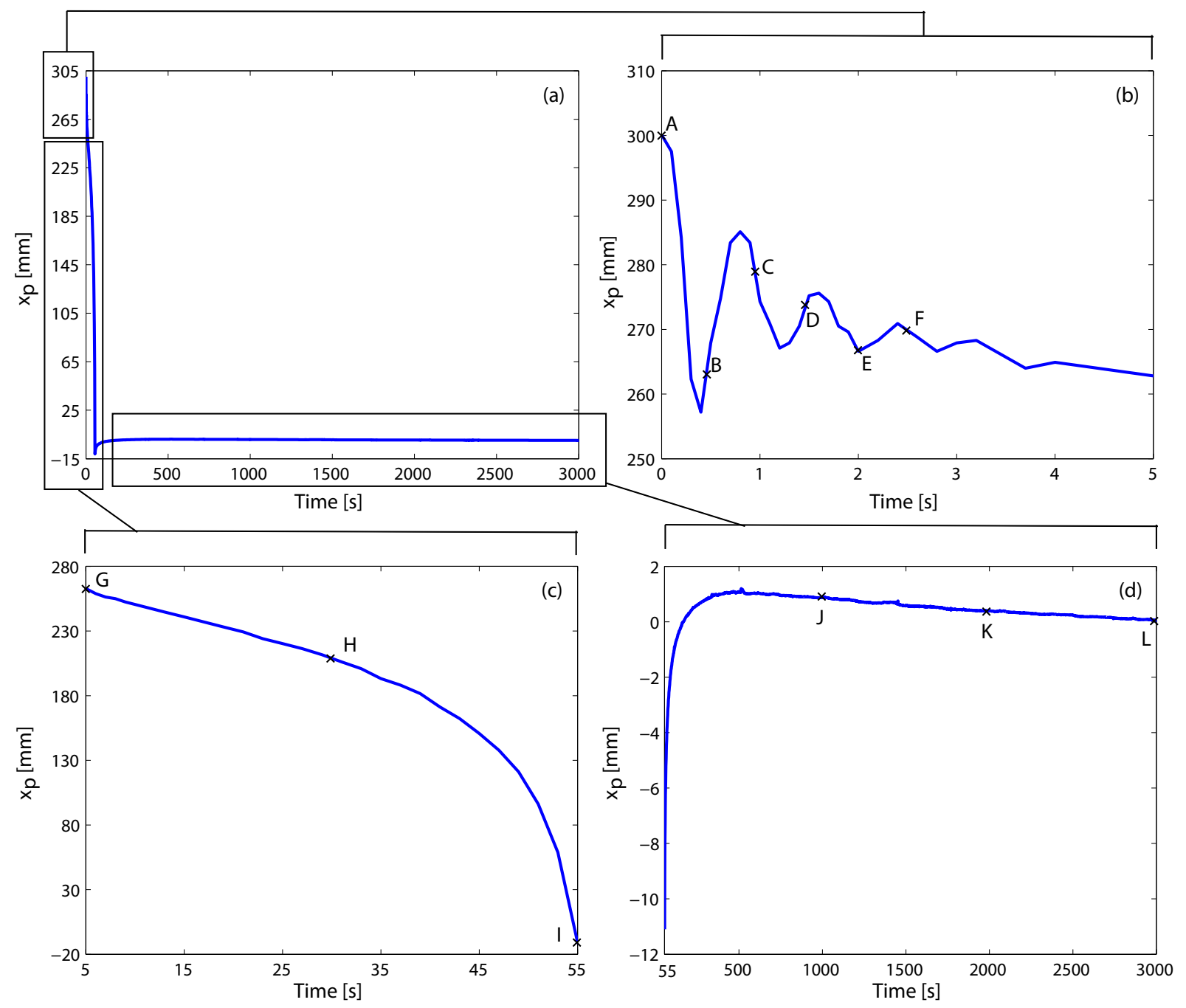

Figure 7: Displacement of point P during deployment. 


\section{A. Review of Linear Viscoelasticity}

The theory of linear viscoelasticity is well established to describe the time and temperature dependence of the mechanical properties of polymers. ${ }^{10-13}$ A Prony series is widely used to describe the linear viscoelastic behavior over a finite range of time scales. The relaxation modulus under isothermal conditions is written as

$$
E(t)=E_{\infty}+\sum_{i=1}^{n} E_{i} e^{-\left(t / \rho_{i}\right)},
$$

where $t$ is time, $E_{\infty}$ is the long term modulus, $E_{i}$ are the Prony coefficients, and $\rho_{i}$ are the relaxation times. The constitutive relation for uniaxial deformation is expressed in the form of the Boltzmann superposition integral,

$$
\sigma(t)=\int_{0}^{t} E(t-\tau) \frac{d \epsilon(\tau)}{d \tau} d \tau
$$

where $\sigma$ is stress and $\epsilon$ is strain.

For the class of thermorheologically simple materials, the effects of time and temperature on the material behavior can be treated in the same manner through the time-temperature superposition principle, ${ }^{14}$

$$
E(t, T)=E\left(t^{\prime}, T_{0}\right), \quad t^{\prime}=\frac{t}{a_{T}(T)},
$$

where $t^{\prime}$ is called the reduced time, $T_{0}$ is the reference temperature, and $a_{T}(T)$ is the temperature shift factor. The time-temperature superposition principle states that the modulus at temperature $T$ and time $t$ is the same as the modulus at a reference temperature $T_{0}$ at a reduced time $t^{\prime}$. Thus, one can relate the viscoelastic behavior at one temperature to that at another temperature by a shift in the time scale. When temperature varies with time, the reduced time is obtained by integration,

$$
t^{\prime}(t)=\int_{0}^{t} \frac{d \tau}{a_{T}(T(\tau))} .
$$

Based on this principle, a master curve can be constructed at an arbitrary reference temperature by shifting the relaxation moduli at any other temperatures to the reference temperature. On a log-log plot of relaxation versus time, this is equivalent to a horizontal shift with a distance of $\log a_{T}(T)$. The resulting master curve is a plot of relaxation modulus on the reduced time scale and describes both the time and temperature dependence of the material behavior.

One commonly used relation for the temperature shift factor of polymers is the empirical WilliamsLandel-Ferry (WLF) equation, ${ }^{15}$

$$
\log a_{T}=-\frac{C_{1}\left(T-T_{0}\right)}{C_{2}+\left(T-T_{0}\right)},
$$

in which $C_{1}$ and $C_{2}$ are material constants that depend on the particular polymer and the logarithm is of base ten.

For isotropic solids the uniaxial constitutive relation in Equation 2 can be generalized to three dimensions by decomposing stress and strain into deviatoric and dilatational components. The corresponding hereditary integrals are written as

$$
\begin{aligned}
s_{i j}(t) & =2 \int_{0}^{t} G(t-\tau) \frac{\partial e_{i j}(\tau)}{\partial \tau} d \tau \\
\sigma_{k k}(t) & =3 \int_{0}^{t} K(t-\tau) \frac{\partial \epsilon_{k k}(\tau)}{\partial \tau} d \tau
\end{aligned}
$$

where $s_{i j}$ and $e_{i j}$ are the deviatoric stress and strain, $\sigma_{k k}$ and $\epsilon_{k k}$ are the dilatational stress and strain, $G$ is the shear modulus and $K$ is the bulk modulus. They are related to uniaxial modulus through

$$
G(t)=\frac{E(t)}{2(1+\nu)},
$$




$$
K(t)=\frac{E(t)}{3(1-2 \nu)},
$$

where $\nu$ is the Poisson's ratio, which is assumed to be constant Poisson's ratio. It has been shown that the assumption of a time independent Poisson's ratio is valid only under the condition of incompressibility ${ }^{16-19}$ therefore a value of 0.49 is used for analysis.

\section{B. Characterization of Low Density Polyethylene}

Linear viscoelastic properties of LDPE were characterized through a series of tensile relaxation tests on rectangular test coupons that had been subjected to the same thermal cycle as during the manufacture of the tape-springs. Specimens with dimensions of $165 \mathrm{~mm}$ by $40.0 \mathrm{~mm}$, a thickness of $0.78 \mathrm{~mm}$ and a density of $930 \mathrm{~kg} / \mathrm{m}^{3}$ were tested inside an environmental chamber. A type-T thermocouple made of Copper/Constantan was attached to the surface of a dummy LDPE specimen close to the test specimen to monitor the actual specimen temperature. As a test for stable temperature conditioning inside the environmental chamber, a temperature impulse was imposed and the subsequent temperature variation over time measured by the built-in thermocouple and the dummy specimen thermocouple were recorded. It was found that the temperature readings from the two thermocouples became identical 30 minutes after the impulse. This indicates that thermal equilibrium can be established within such time frame, and this thermal conditioning time was allowed prior to each test.

Prior to testing, the position of the load frame was adjusted so that a zero axial preload was obtained. To further reduce experimental errors, the specimens were allowed to rest under zero load for 1 hour to ensure the transient effects due to crosshead adjustment had disappeared. Relaxation tests were carried out at temperatures of $0^{\circ} \mathrm{C}, 10^{\circ} \mathrm{C}$ and $22^{\circ} \mathrm{C}$. Test data at low temperatures are required to obtain a close approximation to the instantaneous modulus. At each temperature, test coupons were stretched to a strain of 0.005 in 1 second and held constant for 3 hours. The longitudinal and transverse strains in the specimen were measured using two laser extensometers (Electronic Instrument Research Ltd LE-05) set at a recording rate of $5 \mathrm{~Hz}$.

The relaxation moduli measured are shown in Figure 8. The initial portion of the relaxation test data after loading with a finite strain rate deviates from that in the case of ideal instantaneous straining. It has been demonstrated the difference becomes negligible in about 10 times the loading time. ${ }^{20}$ For this reason, the data obtained during the first $10 \mathrm{~s}$ after loading were discarded. The individual relaxation moduli at $T=0^{\circ} \mathrm{C}$ and $T=10^{\circ} \mathrm{C}$ were shifted to the reference temperature of $T_{0}=22^{\circ} \mathrm{C}$ to form a master curve. The corresponding shift factors were determined so that the shifted relaxation moduli and the unshifted one at $T_{0}$ lie along a single smooth curve. Figure 9 depicts the master curve of LDPE at the reference temperature.

The long term modulus, Prony coefficients and relaxation times were determined by fitting the Prony series representation, Equation (1), to the master curve using the Levenberg-Marquardt optimization algorithm. Table 1 summarizes the Prony series parameters. Similarly, the material constants $C_{1}$ and $C_{2}$ were found by fitting the temperature shift data to the WLF equation (Equation (5)) and the values obtained were

$$
C_{1}=-8.74 \text { and } \quad C_{2}=-40.41
$$

\section{Finite Element Implementation}

Finite element simulations that make use of the viscoelastic material model established in Section III were carried out with the commercial software Abaqus/Standard ${ }^{21}$ to obtain comparisons for the behavior observed in the two types of test. Since the problem under study requires the computation of long term response of a dissipative structure, an implicit simulation code like Abaqus/Standard is suitable because the time increments can be varied according to the degree of nonlinearity encountered and the accuracy desired.

\section{A. Analysis Details}

The viscoelastic material properties summarized in Table 1 were defined with the option *VISCOELASTIC, TIME $=$ PRONY. A user subroutine (UTRS) was written to define the temperature shift factor with Equa- 


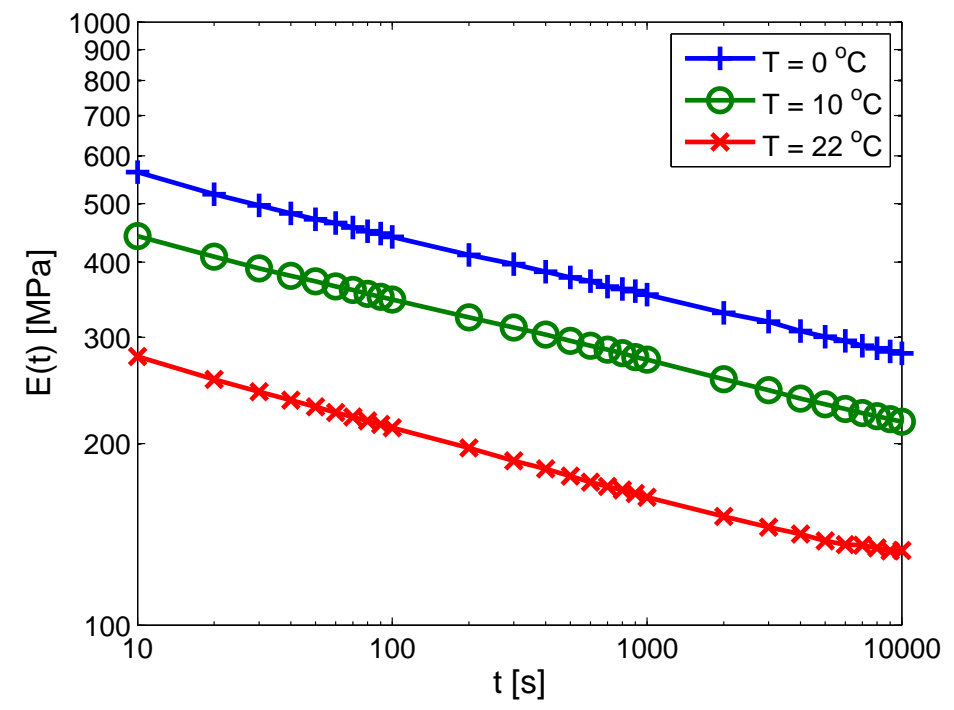

Figure 8: Relaxation moduli versus time at different temperatures.

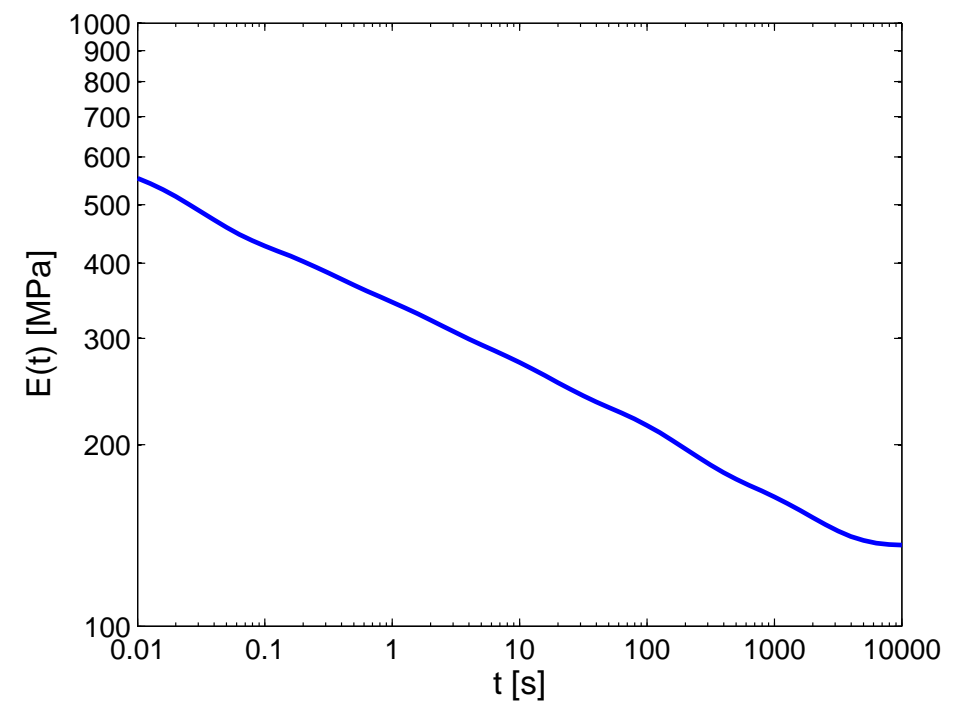

Figure 9: Master curve for LDPE at $22^{\circ} \mathrm{C}$. 


\begin{tabular}{c|c|c}
\hline \hline$i$ & $E_{i}[M P a]$ & $\rho_{i}[\mathrm{~s}]$ \\
\hline$\infty$ & 136.2 & - \\
1 & 150.6 & $2.43 \times 10^{-2}$ \\
2 & 74.81 & $2.17 \times 10^{-1}$ \\
3 & 68.26 & 1.52 \\
4 & 65.22 & $1.24 \times 10$ \\
5 & 62.85 & $1.49 \times 10^{2}$ \\
6 & 49.83 & $1.69 \times 10^{3}$ \\
\hline \hline
\end{tabular}

Table 1: Prony series parameters for LDPE.

tion 5. With this general capability, the change in material modulus within the structure due to temperature gradient and history can be readily accounted for.

Simulations of the quasi-static folding and stowage test in Section II.A were carried out with a model including 6800 quadrilateral shell elements (S4) with a maximum dimension of $2 \mathrm{~mm}$ as shown in Figure 10. The mesh density in the circumferential direction was twice that in the longitudinal direction for more precise computation of the localized fold. Displacement boundary conditions were defined for two nodes, one on each end section of the tape-spring and coinciding with the mid-point of the cross section. All the translational degrees of freedom of the bottom node were constrained and gravity load was prescribed throughout the analysis.

A quasi-static analysis was carried out in two steps, as follows. During the folding step, a downward displacement of $80 \mathrm{~mm}$ was imposed on the top node, at the same rates and temperatures used in the experiments. In the stowage step, the degrees of freedom of the top node were held constant for $5000 \mathrm{~s}$ and equal to their values at the end of the folding step.

The accuracy of integration during the quasi-static steps was controlled by specifying the tolerance parameter through the command ${ }^{*} \mathrm{CETOL}$, which puts a limit on the maximum change in creep strain rate allowed over a time increment. A value of $1 \times 10^{-4}$ was found to be adequate for obtaining accurate solutions.

Simulations of the dynamic deployment test in Section II.B were carried out with a similar mesh, but with 2500 elements and a maximum dimension of $4 \mathrm{~mm}$. For this simulation, the bottom end section of the tape-spring was fixed and a lateral displacement of $300 \mathrm{~mm}$ was imposed over $9 \mathrm{~s}$ to the top node, to simulate folding. The boundary conditions on the top node were instantaneously removed after a stowage step of $983 \mathrm{~s}$. The analysis was quasi-static for these first two steps and dynamic after the removal of the top node constraint. The deployment process was simulated for $3000 \mathrm{~s}$.

\section{B. Modeling of Geometric Imperfections}

The behavior of thin shells is known to be sensitive to geometric imperfections from sources such as manufacturing and load misalignment: if such effects are not considered the response of a structure usually appears much stiffer than observed in experiments. This was the case for initial simulation attempts of thin tape-springs assuming perfect geometry, until two major imperfections were identified and appropriately modeled.

The first imperfection considered was the uneven distribution of thickness resulting from the fabrication process. To account for this defect, a rectangular grid was drawn on the tape-spring and the actual thickness at the grid points was measured with a coating thickness gauge (Elcometer 456) with a resolution of 10 microns. The grid spacing is such that the distance between adjacent grid points is $16 \mathrm{~mm}$ along the length and $10 \mathrm{~mm}$ along the circumference of the tape-spring. The thickness distribution was included in the finite element model by specifying the element thickness to be calculated from the nodal thicknesses. Since the mesh is much finer than the thickness measurement grid, spline interpolation was used to determine the thickness at all node positions. Figure 11 shows the thickness contours of a tape-spring specimen.

Another imperfection found to be important was the residual deformation resulting from previous loading cycles, due to residual viscoelastic deformation. Complete shape recovery, in particular of the cross section geometry in the fold region, requires several weeks to occur and hence a small amount of deformation 


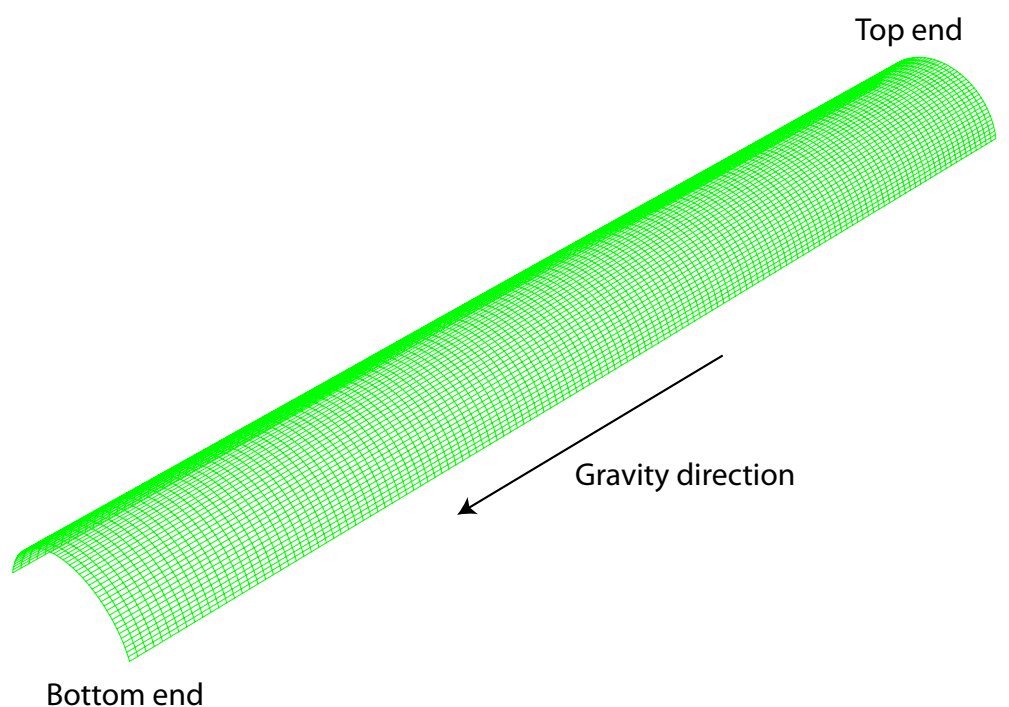

Figure 10: Finite element model of tape-spring.

remained whenever a test was repeated. To characterize this imperfection, the residual deformed shape was estimated from simulations of a perfect tape-spring. This was done by first performing the folding and stowage analysis described previously, and subsequently allowing the tape-spring to freely recover. The nodal displacements at the end of the recovery period were then seeded as a perturbation to the perfect tape-spring geometry and thus generate an imperfect initial shape for the actual analysis.

The magnitude of this perturbation was defined by measuring the mid-section of the specimen at three points using laser sensors prior to testing and the imperfections were chosen so that the values at these points were matched. Figure 12 shows the contour plot of a particular residual displacement distribution used for constructing the imperfect initial shape and the locations at which displacement measurements were taken.

\section{Comparison of Results and Discussions}

The load vs. time profiles from the experiments and the simulations, and for two different temperatures are compared in Figure 13. Overall, the simulations show good agreement with the observed response. However, the simulation slightly overpredicts the peak load, by $0.9 \mathrm{~N}$ and $0.3 \mathrm{~N}$ for temperatures of $15^{\circ} \mathrm{C}$ and $22^{\circ} \mathrm{C}$ respectively. The effect of such discrepancy on the long term response of the structure is negligible because it only lasts a few seconds. The error between simulated and experimental results has become insignificant after the the fold has completely formed at about $7 \mathrm{~s}$. This can be seen more clearly in Figure 14, where the load in the stowed configuration is plotted on a semi-log scale. The load relaxation behavior is well captured by the simulation.

Figure 15 compares the lateral displacement of tape-spring, $x_{p}$, during deployment. The general features of the response have been reproduced by the simulations, but there are also some noticeable discrepancies. The period of vibration is about $0.3 \mathrm{~s}$ longer in the simulated response during the initial dynamic phase shown in Figure 15(a), but the oscillations are about the same mean displacement in experiments and simulations. The largest discrepancy is found in the quasi-static recovery phase shown in Figure 15(b), where the simulation overpredicts the overshoot by $20 \mathrm{~mm}$, occurring about $35 \mathrm{~s}$ earlier than in the experiment. Dynamic response is also observed in the simulation right after the overshoot, but is not seen in the experiments. Nonetheless, the long term creep recovery is reasonably well predicted, as shown in Figure 15(c). It is believed that the main reason for the discrepancies is thermal variations in the experimental environment, which would require experiments conducted inside a chamber with temperature stabilization.

The effects of imperfections have also been examined by comparing simulations of perfect and imperfect tape-springs. The peak load is highly sensitive to both thickness distribution and residual deformation and is significantly lower in imperfect tape-springs. It is also found that even a small residual deformation reduces the time required for a complete fold to form. However, it has little effect on deployment provided that 


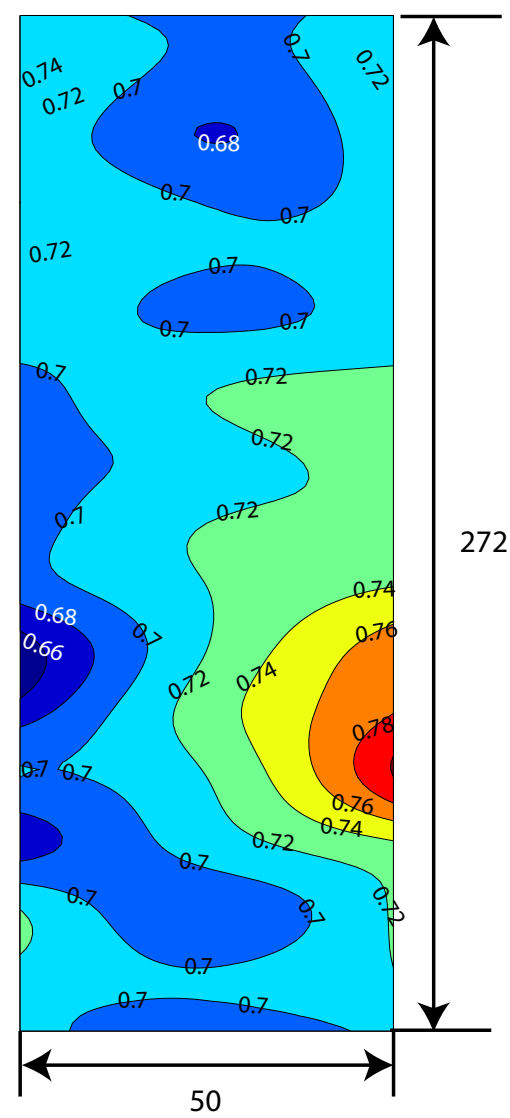

Figure 11: Thickness distribution, in millimeters, of tape-spring specimen. The horizontal coordinate is the cross-sectional arc-length. 


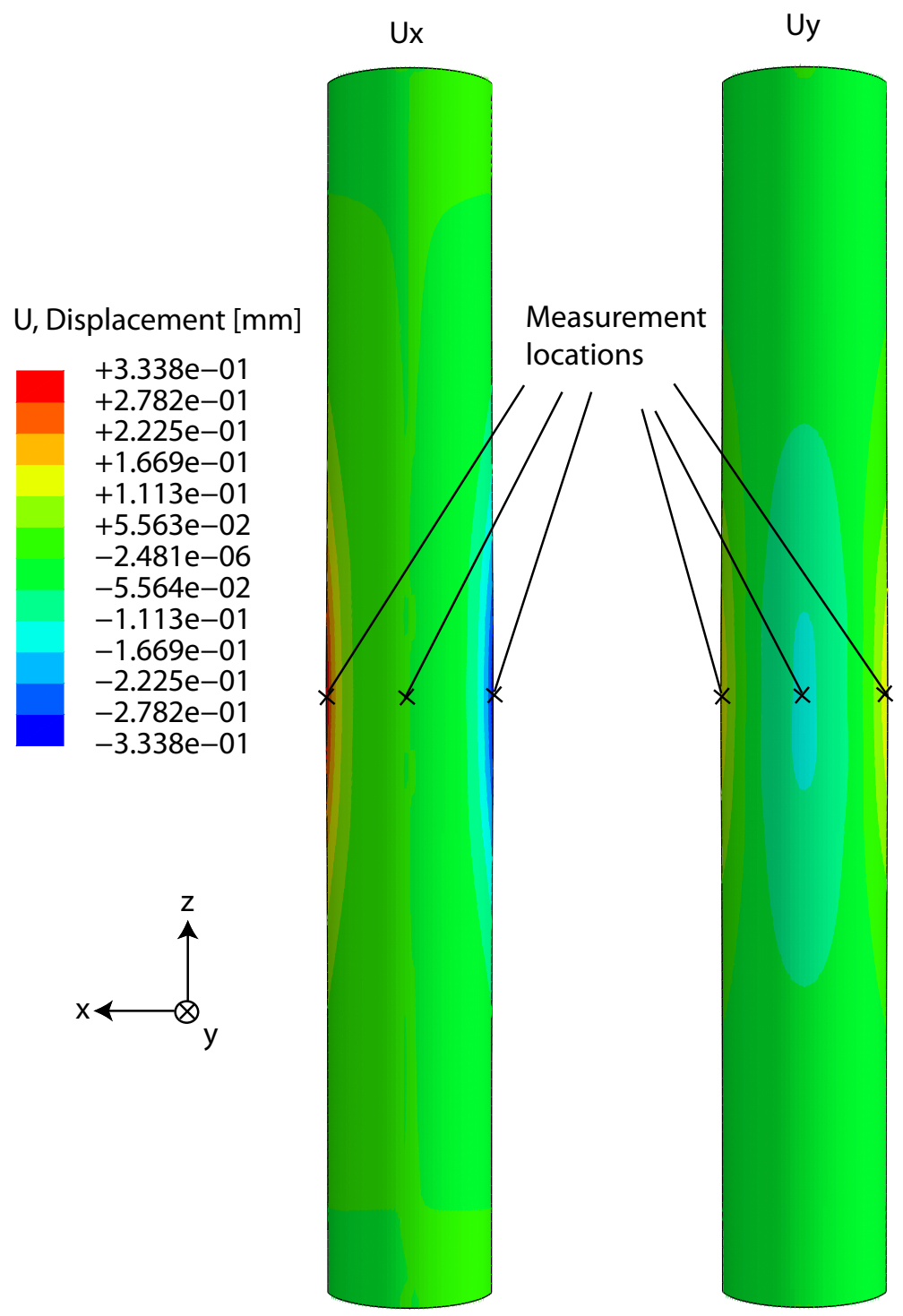

Figure 12: Residual displacements for generating imperfections in tape-spring geometry. 
sufficient stowage time is allowed.

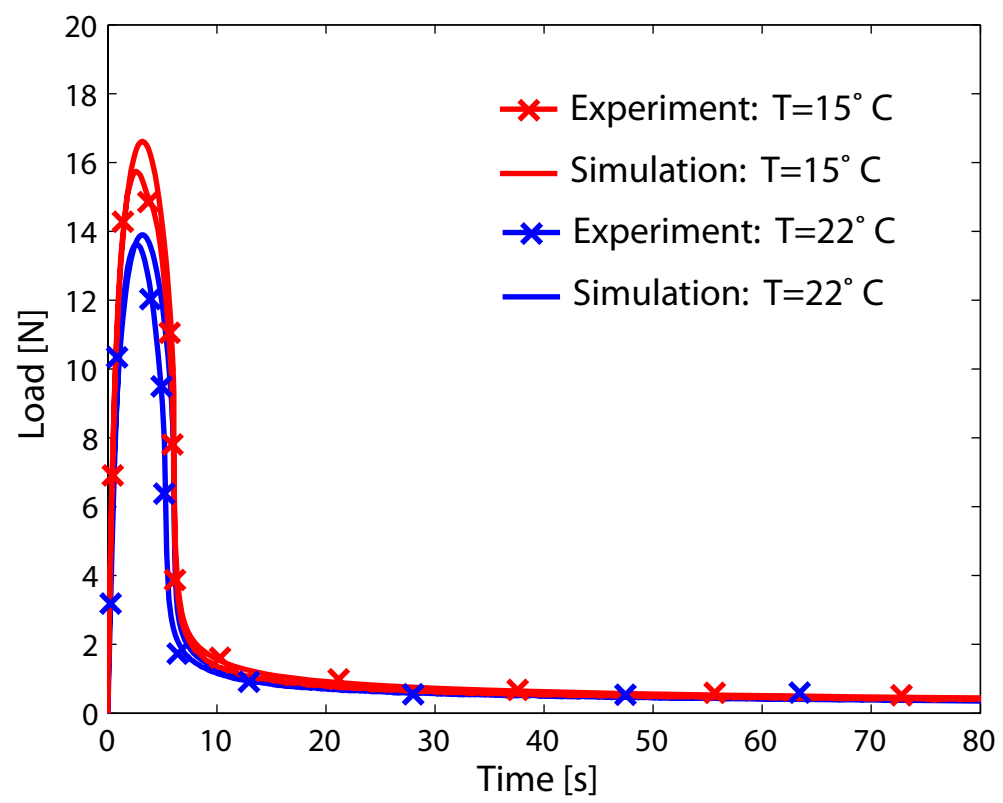

Figure 13: Comparison of load vs. time response during folding, for a displacement rate of $1 \mathrm{~mm} / \mathrm{s}$.

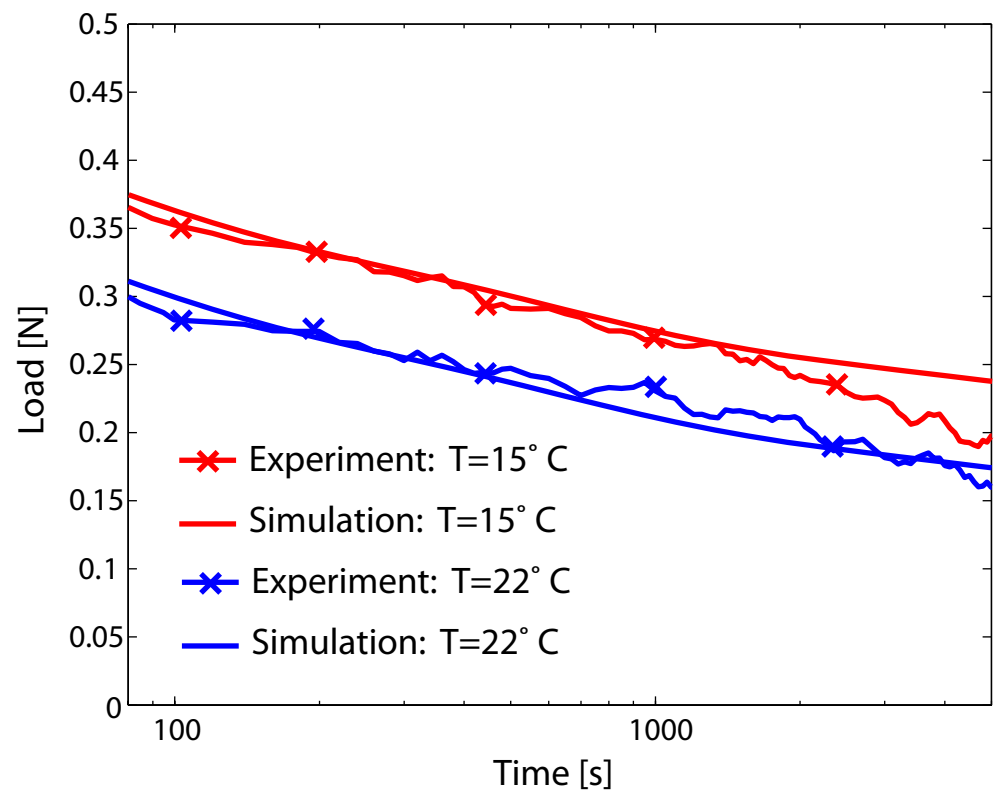

Figure 14: Comparison of load relaxation vs. time during stowage.

\section{Conclusions}

The present study is the first attempt to tackle the entire folding, stowage, and deployment process of a viscoelastic tape-spring as a continuous time-dependent event. It has been shown that the peak load during folding rises with decreasing temperature and increasing folding rates. The folding and stowage process is characterized by significant load relaxation. It has also been shown that establishing the correct load and 

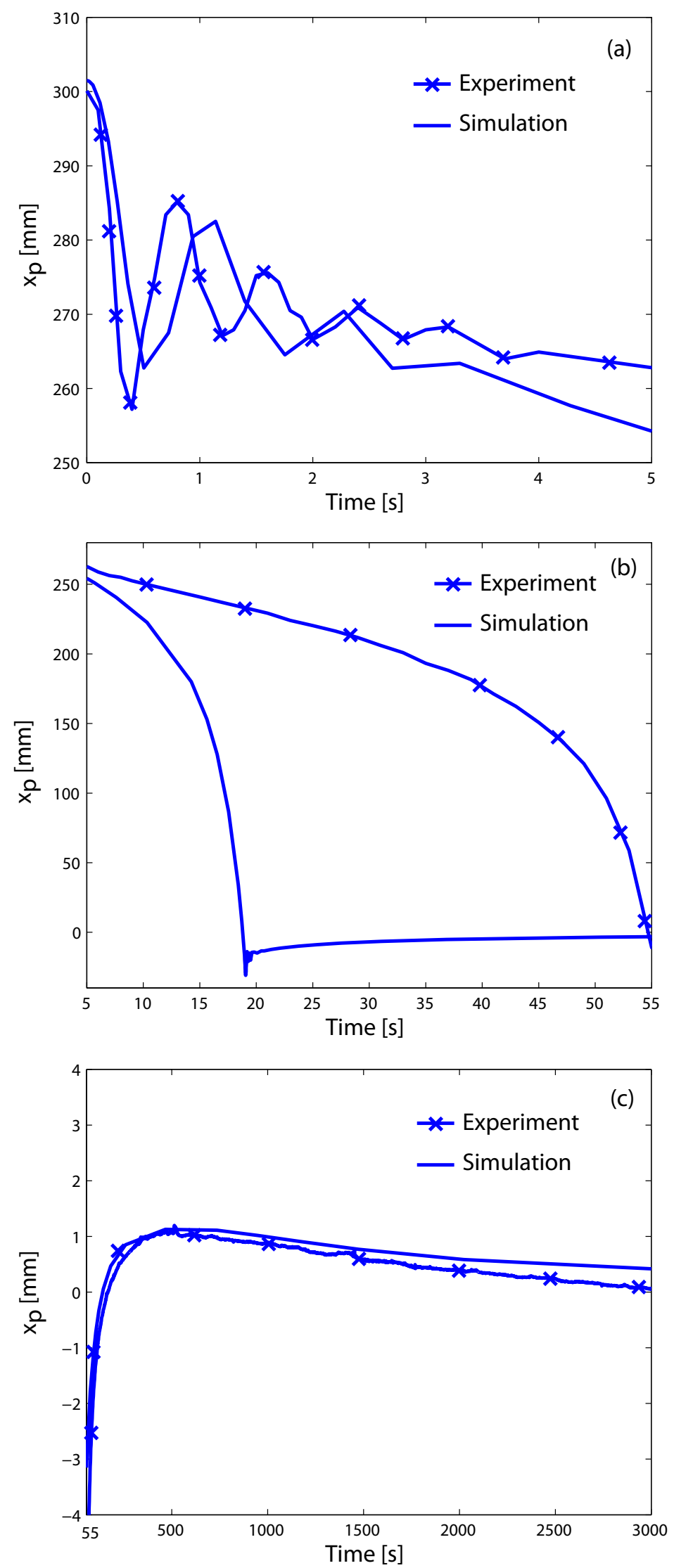

Figure 15: Comparison of lateral displacement of tape-spring free end: (a) transient dynamic response, (b) quasi-static deployment and (c) long term creep recovery.

\section{6 of 17}


deformation history is important for understanding the subsequent deployment process.

Deployment begins with a weak dynamic response accompanied by a low magnitude vibration. This short dynamic phase is followed by a quasi-static deployment that returns the tape-spring to almost the straight deployed configuration, but with a slight overshoot. The full recovery requires a final slow creep recovery process. An interesting observation is that the fold location stays constant during deployment, as opposed to the moving fold seen in elastic tape-springs. It is speculated that locking of the fold is due to local stiffness reduction at the fold after relaxation. This particular deformed configuration becomes a stable equilibrium, while only neutral equilibrium is attained for elastic tape-springs.

Finite element simulations based on linear isotropic viscoelasticity and measured material properties have been developed to simulate the behavior of tape-springs. It has been demonstrated that the simulation techniques presented are capable of capturing all the details for folding and stowage behavior at different temperatures and rates. The key features of dynamic behavior are also reproduced, but with some discrepancy that needs further investigation. The finite element implementation has general capabilities that can handle varying temperature gradients and histories for viscoelastic deployable structures in both quasi-static and dynamic situations.

\section{Acknowledgment}

The authors are grateful to Professor Wolfgang Knauss for helpful comments and discussions. Financial support for KK by the Croucher Foundation is acknowledged.

\section{References}

\footnotetext{
${ }^{1}$ Yee, J.C.H., Pellegrino, S., "Composite tube hinges," Journal of Aerospace Engineering, Vol. 18, 2005, pp. $224-231$.

${ }^{2}$ Adams, D.S., and Mobrem, M., "Lenticular jointed antenna deployment anomaly and resolution onboard the Mars Express Spacecraft," Journal of Spacecraft and Rockets, Vol. 46, No. 2, 2009, pp. 403-408.

${ }^{3}$ Mobrem, M., and Adams, D.S., "Deployment analysis of lenticular jointed antennas onboard the Mars Express Spacecraft," Journal of Spacecraft and Rockets, Vol. 46, No. 2, 2009, pp. 394-402.

${ }^{4}$ Domber, J.L., Hinkle, J.D., Peterson, L.D., and Warren, P.A., "Dimensional repeatability of an elastically folded composite hinge for deployed spacecraft optics," Journal of Spacecraft and Rockets, Vol. 39, No. 5, 2002, pp. 646-652.

${ }^{5}$ Soykasap, O., "Deployment analysis of a self-deployable composite boom," Composite Structures, Vol. 89, 2009, pp. 374381.

${ }^{6}$ Kwok, K., and Pellegrino, S., "Shape recovery of viscoelastic deployable structures," 51st AIAA/ASME/ASCE/AHS/ASC Structures, Structural Dynamics, and Materials Conference, AIAA, Orlando, Florida, April 2010.

${ }^{7}$ Soykasap, O., Pellegrino, S., Howard, P., and Notter, M., "Folding Large Antenna Tape Spring," Journal of Spacecraft and Rockets, Vol. 45, No. 3, 2008, pp. 560-567.

${ }^{8}$ Capodagli, J., and Lakes, R.S., "Isothermal viscoelastic properties of PMMA and LDPE over 11 decades of frequency and time: a test of time-temperature superposition," Rheologica Acta, Vol. 47, 2008, pp. 777-786.

${ }^{9}$ Seffen, K.A., and Pellegrino, S., "Deployment dynamics of tape springs," Proceedings of the Royal Society of London, Seris A: Mathematical and Physical Sciences, Vol. 455, 1999, pp. 1003-1048.

${ }^{10}$ Coleman, B.D., Noll, W., "Foundations of linear viscoelasticity," Reviews of Modern Physics, Vol. 33, 1961, pp. $239-249$.

${ }^{11}$ Christensen, R.M., Theory of Viscoelasticity: An Introduction, 2nd ed., Academic Press, New York, 1982.

${ }^{12}$ Flugge, W., Viscoelasticity, Springer-Verlag, New York, 1975.

${ }^{13}$ Tschoegl, N.W., The Phenomenological Theory of Linear Viscoelastic Behavior, Springer-Verlag, Heidelberg, 1989.

${ }^{14}$ Ferry, J.D., Viscoelastic Properties of Polymers, 3rd ed., John Wiley and Sons, New York, 1980.

${ }^{15}$ Williams, M.L., Landel, R.F., and Ferry, J.D., "The temperature dependence of relaxation mechanisms of amorphous polymers and other glass-forming liquids," Journal of the American Chemical Society, Vol. 77, 1955, pp. 3701-3707.

${ }^{16}$ Hilton, H.H., "Implications and constraints of time-independent Poisson ratios in linear isotropic and anisotropic viscoelasticity," Journal of Elasticity, Vol. 63, 2001, pp. 221-251.

${ }^{17}$ Hilton, H.H., and Yi, S., "The significance of (an)isotropic viscoelastic Poisson ratio stress and time dependencies," International Journal of Solids and Structures, Vol. 35, 1998, pp. 3081-3095.

${ }^{18}$ Lakes, R.S., "On Poisson's ratio in linearly viscoelastic solids," Journal of Elasticity, Vol. 85, 2006, pp.45-63.

${ }^{19}$ Tschoegl, N.W., Knauss, W.G., and Emri, I., "Poisson's ratio in linear viscoelasticity - a critical review," Mechanics of Time-Dependent Materials, Vol. 6, 2002, pp.3-51.

${ }^{20}$ Lee, S., and Knauss, W.G., "A note on the determination of relaxation and creep data from ramp tests," Mechanics of Time-Dependent Materials, Vol. 4, 2000, pp. 1-7.

${ }^{21}$ Abaqus/Standard, Software, Ver. 6.9, Simulia, Providence, R.I., 2009
} 